Original Paper

\title{
Structure Determination of Naproxen Photoproducts in the Tablet Generated by the UV Irradiation
}

\author{
Kohei KAWABATA*1, Yoshie MIZUTA ${ }^{1}$, Kana ISHIHARA ${ }^{1}$, Ayaka TAKATO ${ }^{1}$, Sayaka OSHIMA ${ }^{1}$, \\ Shiori AKIMOTO ${ }^{2}$, Masanori INAGAKI ${ }^{1}$, Hiroyuki NISHI ${ }^{1}$ \\ ${ }^{1}$ Faculty of Pharmacy, Yasuda Women's University. 6-13-1 Yasuhigashi, Asaminami-ku, Hiroshima 731-0153, Japan \\ ${ }^{2}$ Graduate School of Biomedical and Health Sciences, Hiroshima University, 1-2-3 Kasumi, Minami-ku,
}

Hiroshima 734-8553, Japan

\begin{abstract}
Change of the dosage form of the medicine is a useful method for the improvement of the medicine-taking compliance. However, the photostability of the medicine may be decreased on account of the change of the dosage form, followed by the change of the quality of it. On the other hand, there is few information focused on the photostability of the dosage-changed medicine. In this study, the effects of the change of the dosage form on the photostability of some non-steroidal anti-inflammatory drugs (NSAIDs) are investigated. Photo-exposure by means of the black light, containing ultraviolet (UV) at mainly $365 \mathrm{~nm}$, induced the change of the color of naproxen tablets although the content of the active compound monitored by the high-performance liquid chromatography (HPLC) was not changed. However, the change of the dosage form of naproxen tablets to the powder and the suspension induced the change of the photostability followed by the decrease of the content of the active compound and the generation of two photoproducts. By means of the nuclear magnetic resonance (NMR) analysis, structures of two naproxen photoproducts were determined as 2-acetyl-6-methoxy-naphthalene and 1-(6-methoxy-2-naphthyl) ethanol. This is the first report evaluated the generation of naproxen photoproducts induced by the long-wavelength UV irradiation in the formulation.
\end{abstract}

Keywords: Naproxen; Photostability; Dosage-form; Photodegradation; Photoproducts

\section{Introduction}

Stability evaluation of the new medicine is required for the new drug application in addition to the evaluation of effectiveness and safety. To evaluate the stability, the Stability Test Guidelines agreed with the International Conference on Harmonization of Technical Requirements for Registration of Pharmaceuticals for Human Use (ICH) have been established (ICH-Q1A, Stability testing of new drug substances and products), and it prescribed the test methods such as long-term storage test (under the conditions of $25 \pm 2{ }^{\circ} \mathrm{C}$ and $60 \pm 5 \% \mathrm{RH}$ ), accelerated test and severe test (under the condition of $40 \pm 2{ }^{\circ} \mathrm{C}$ and $75 \pm 5 \% \mathrm{RH}$ ) [1]. Severe test is required for the evaluation of the change of the quality of the medicine induced by the heat, humidity

${ }^{*}$ Corresponding author: Kohei KAWABATA

Tel: +81-82-878-9440; Fax: +81-82-878-9540

E-mail: kawabata-k@yasuda-u.ac.jp and photo-exposure. In the case of the photostability evaluation, it is also necessary to conduct tests based on the guidelines for the photostability evaluation (ICH-Q1B, Photostability testing of new drug substances and products) agreed with ICH [2]. It is important to investigate the effect of the photo-exposure on the medicine for the safety use of it.

There are various reports focused on the photodegradation of medicines, such as calcium-channel blocker barnidipine and nifedipine, diuretic drug furosemide and non-steroidal anti-inflammatory drugs (NSAIDs) diclofenac induced by the photo-exposure [3-6]. Our previous reports showed that photo-conversion of sulindac, which is one of the NSAIDs, to trans-sulindac [7].

Received: 27 July 2019

Accepted: 16 September 2019

J-STAGE Advance Published: 24 September 2019

DOI: 10.15583 /jpchrom.2019.020 
It is possible that photodegradation of the medicine induces the generation of the photoproduct, which may have another pharmacological activity, in addition to the decrease of the effectiveness due to the degradation of active compound. Photodegradable medicines are sold and used after the formulation to the dosage form (tablet, capsule, powder and so on), which is based on these information, for masking the effect of the photo-exposure.

Most of the study investigating the photodegradation of medicines have been carried out using the solution due to that photochemical reaction using the solution is more active than using the solid-form medicine. In the case of the solution, test medicines are tending to be degradable for the photo-exposure following to the conversion to various photoproducts. This phenomenon can cause the inaccurate estimation of the photostability of the solid-form medicine, but there are few reports evaluating the photostability of the solid-form medicines. Interview form published from the maker shows the results of stability tests, but they have no data focused on the photostability of the medicines which are crushed and suspended.

In this study, we evaluated the photostability of the solid-form medicine at first. Photo-exposure to eight NSAIDs tablets (aspirin, acetaminophen, ibuprofen, etodolac, diclofenac, naproxen, meloxicam and loxoprofen) were demonstrated, and the residual rate of the active compound was monitored by means of high-performance liquid chromatography (HPLC). Moreover, the change of the dosage-form on the photostability of naproxen tablet was evaluated. Due to the generation of two photoproducts of naproxen induced by the ultraviolet (UV) irradiation, chemical structures of them were determined by means of nuclear magnetic resonance (NMR) analysis. This is the first report showing the generation of naproxen photoproducts in the formulation by the UV irradiation.

\section{Experimental}

\subsection{Chemicals}

Aspirin tablets, acetaminophen tablets, etodolac tablets, diclofenac tablets, meloxicam tablets, loxoprofen tablets were purchased from Towa Pharmaceutical Corporation (Osaka, Japan), ibuprofen tablets were purchased from Tatsumi Kagaku Corporation (Kanazawa, Japan), naproxen tablets were purchased from Mitsubishi Tanabe Pharma Corporation (Osaka, Japan). Chloroform- $d 4$ ( $d$, 99.8\%) containing tetramethyl silane $(0.05 \%, v / v)$, methanol, acetic acid and naproxen drug substance were purchased from Fujifilm Wako Pure Chemical Corporation (Osaka, Japan). Milli-Q $(18.2 \Omega / \mathrm{cm})$ water was prepared by using a Milli-Q water purification system (Merck, Darmstadt, Germany).

\subsection{Irradiation experiments}

The photo-exposure was carried out in the light cabinet
20W FL20S BLB black light lamp (Toshiba, Tokyo, Japan). This lamp irradiates the UV mainly at $365 \mathrm{~nm}$, which is contained in the sunlight, and irradiation intensity was 500 $\mu \mathrm{W} / \mathrm{cm}^{2} / \mathrm{sec}(365 \mathrm{~nm})$ measured using a digital radiometer with $365 \mathrm{~nm}$ sensor (UVX-36, UVP, Upland, CA, USA). Tablets were taken out from the press through package (PTP) sheet and exposure to the black light lamp for $24 \mathrm{hr}$ at a $20{ }^{\circ} \mathrm{C}$. In the case of naproxen tablets, tablets were crushed, or suspended to $100 \mathrm{~mL}$ Milli-Q water (sonication for $5 \mathrm{~min}$ after the addition of naproxen tablets to Milli-Q water) for the evaluation of the effects of the change of the dosage form. Prepared powder and suspension were irradiated to the black light as the same as naproxen tablets. Control samples were prepared for the same condition but covered with the aluminum foil to interrupt the UV irradiation.

Irradiated tablet and powder by the UV were suspended in $100 \mathrm{~mL}$ of $50 \%(\mathrm{v} / \mathrm{v})$ methanol exactly and sonicated for $5 \mathrm{~min}$ for the extraction. Residue was removed by the filtration using a membrane filter $(0.45 \mu \mathrm{m}$, Merck $)$ and the filtrate was used for the HPLC analysis. In the case of the suspension sample, methanol was added to make $200 \mathrm{~mL}$ exactly. After that, the residue was removed as the same in the tablet and powder samples, and the filtrate was used for the HPLC analysis.

\subsection{HPLC analysis and UV spectrum analysis}

The HPLC analysis for monitoring the decrease of active compounds was performed on Prominence HPLC system, which is composed of a LC-20AB pump, a SIL-20AC auto-sampler, a SPD-M20A photodiode array detector (PDA) with a LCsolution software, a CBM-20A system controller, a DGU-20A3 degasser, and CTO-20A column oven (Shimadzu Corporation, Kyoto, Japan). The analytical column was a Shim-pack VP-ODS column (5 $\mu \mathrm{m}, 4.6$ x 150 $\mathrm{mm}$, Shimadzu Corporation) and kept at $40{ }^{\circ} \mathrm{C}$ during analysis. Isocratic separations of each sample were achieved using a mobile phase consisting of methanol-water (5:5 (v/v) for ibuprofen, naproxen and loxoprofen; 7:3 (v/v) for aspirin, diclofenac, etodolac, meloxicam; 1:9 (v/v) for acetaminophen) containing acetic acid $(0.1 \%$, v/v). Flow rate was maintained at $1.0 \mathrm{~mL} / \mathrm{min}$. The injection volume was $20 \mu \mathrm{L}$. Retention times of each active compound were as follows; $2.6 \mathrm{~min}$ (aspirin), $7.6 \mathrm{~min}$ (acetaminophen), 10.3 min (ibuprofen), $8.4 \mathrm{~min}$ (etodolac), $9.1 \mathrm{~min}$ (diclofenac), $20.7 \mathrm{~min}$ (naproxen), $8.9 \mathrm{~min}$ (meloxicam), $12.6 \mathrm{~min}$ (loxoprofen). Results of the HPLC analysis were shown as the residual rate of active compounds for amounts of active compounds before the UV irradiation.

The UV absorption spectra of naproxen and its photoproducts were recorded with the PDA detector. The solution containing the UV-irradiated naproxen was injected to the HPLC system and the UV spectra of the peaks of 
naproxen and its two photoproducts were analyzed. Absorption-maximum wavelength ( $\lambda \max )$ of each peak was obtained from these results.

\subsection{Structure determination of naproxen photoproducts}

The photodegradation of the naproxen standard solution was demonstrated for the structure determination of two photoproducts due to same photoproducts were detected from the naproxen medicine (tablet, powder and suspension) and standard solution of naproxen after the UV irradiation. Naproxen drug substance was initially dissolved in methanol $(10 \mathrm{mg} / \mathrm{mL})$ and this solution was added to Milli-Q water for the preparation of the test sample (20 $\mu \mathrm{g} / \mathrm{mL}, 100 \mathrm{~mL}$ ) in the glass vessel (10 samples) by the UV lamp (water depth $3 \mathrm{~cm}$, distanced from lamp source about $15 \mathrm{~cm}$ ). Irradiation times were $3 \mathrm{hr}$ at $20{ }^{\circ} \mathrm{C}$. The UV-irradiated naproxen solution was injected to the HPLC system as the same condition in 2.3. for monitoring of the decrease of naproxen and the generation of its photoproducts. After that, irradiated samples were condensed by the solid phase extraction using cartridges (Oasis HLB cartridge 3cc, Waters Corporation Milford, MA, USA) in a multiple-sample concentration system (Varian Sample Preparation Vac Elute SPS 24, Agilent Technologies, Santa Clara, CA, USA). Samples were eluted using methanol $(3 \mathrm{~mL})$, and all elution were collected and concentrated under the reduced pressure. Residual substances were dissolved in methanol $(1 \mathrm{~mL})$ followed by the HPLC analysis. Two naproxen photoproducts (photoproduct 1, retention time was $17.4 \mathrm{~min}$; photoproduct 2, retention time was $12.8 \mathrm{~min}$ ) were isolated using HPLC repeatedly (about 40 times), and methanol solutions of the collected samples were removed by the evaporation. Two samples were applied to the Oasis HLB cartridge $3 \mathrm{cc}$ and eluted by methanol $(3 \mathrm{~mL})$, followed by the removal of methanol under the reduced pressure. Residual substances were dissolved in chloroform- $d 4$ containing tetramethyl

Table 1. Residual rate of active compounds in tablets after the UV irradiation for $24 \mathrm{hr}$.

\begin{tabular}{cc}
\hline Active compounds & Residual rate (\%) \\
\hline Aspirin & $103.64 \pm 4.24$ \\
Acetaminophen & $98.99 \pm 3.90$ \\
Ibuprofen & $101.33 \pm 3.66$ \\
Etodolac & $98.14 \pm 7.09$ \\
Diclofenac & $99.22 \pm 5.69$ \\
Naproxen & $103.32 \pm 1.58$ \\
Meloxicam & $100.83 \pm 2.38$ \\
Loxoprofen & $108.48 \pm 3.80$ \\
\hline
\end{tabular}

Values are mean \pm S.D. $(n=3)$, The means are not significantly different in Tukey's test $(p<0.05)$. silane for the NMR analysis. The results of NMR analysis were shown in supplemental figures (Fig. A. 1-6).

\subsection{Statistical analysis}

Data are expressed as mean \pm standard deviation (S.D.). The homogeneity of variance was established using a one-way ANOVA. Statistical significance between two groups was estimated by the Student's t-test, and between more than three groups was estimated by Tukey's test. The threshold for assessing significance were $p<0.05$ or $p<$ 0.001 vs control.

\section{Results and discussion}

\subsection{The effect of the UV irradiation on the tablet}

At first, the effects of the UV irradiation on eight NSAIDs tablets were evaluated. Residual rates of active compounds of eight medicines after the UV irradiation were
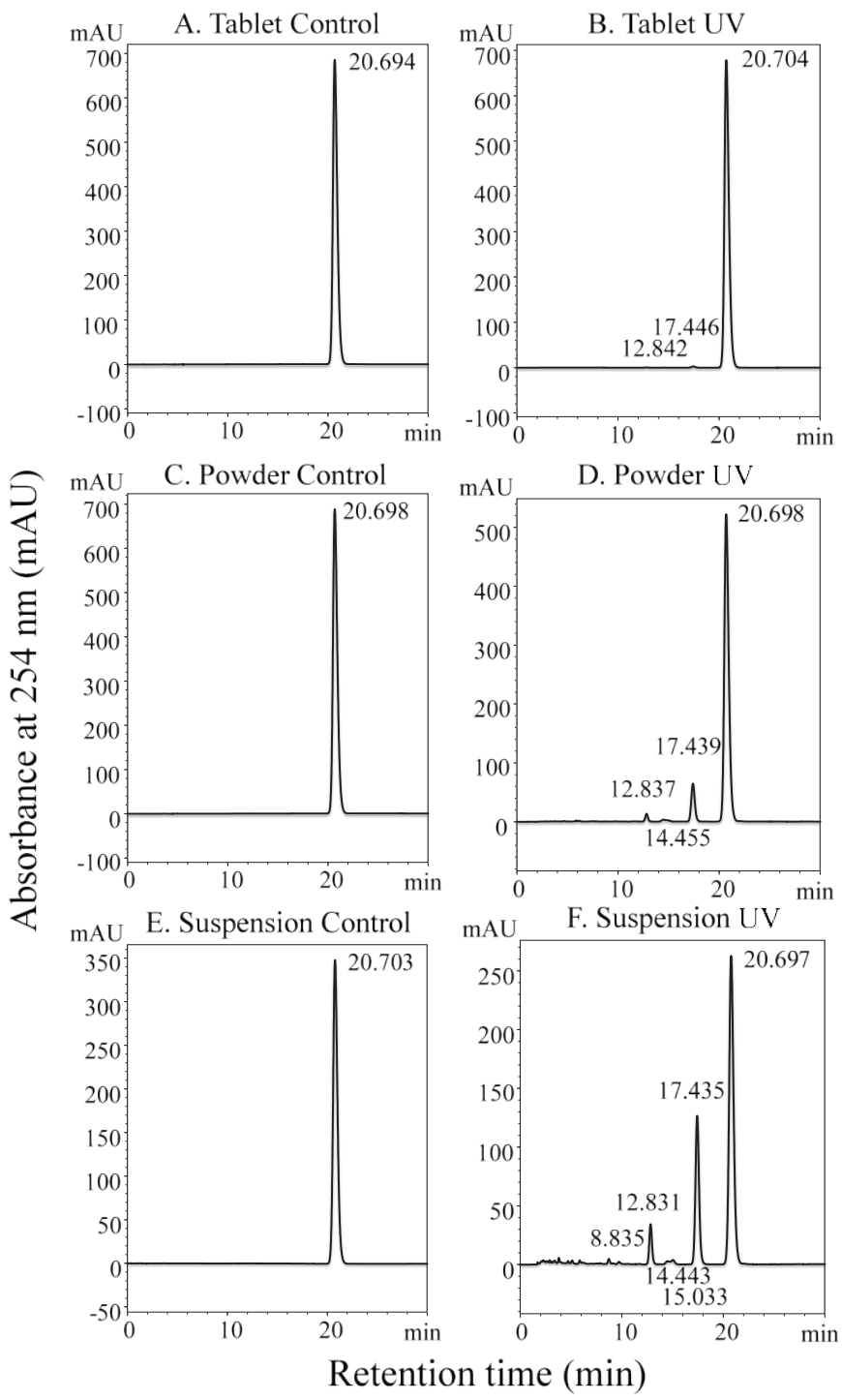

Fig. 1. HPLC analysis of Naproxen tablets and its powder and suspension before and after the UV irradiation for $24 \mathrm{hr}$. (A) Tablet, (B) UV-irradiated tablet, (C) powder, (D) UV-irradiated powder, (E) suspension, (F) UV-irradiated suspension. Detection wavelength: $254 \mathrm{~nm}$. 


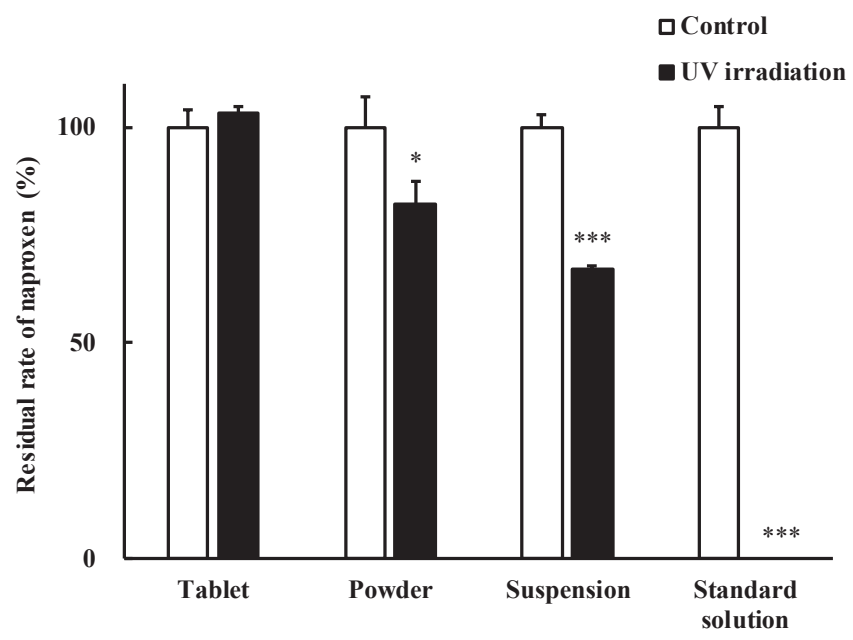

Fig. 2. Destabilization of naproxen in the medicine for the UV irradiation induced by the change of dosage form. Values represent means \pm S.D. $(\mathrm{n}=3-4)$. ${ }^{*}$ Difference compared with control $(P<0.05) .{ }^{* * *}$ Difference compared with control $(P<$ $0.001)$.
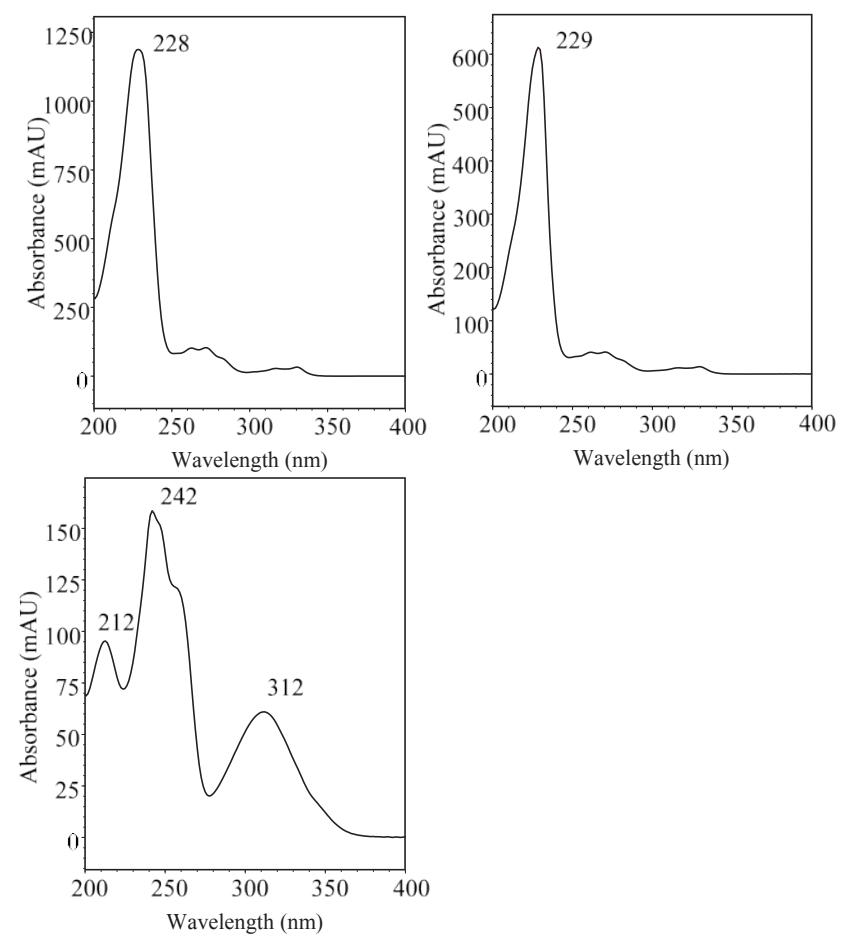

Fig. 3. The UV absorption spectrum of (A) naproxen, (B) photoproduct 1 and (C) photoproduct 2.

shown in Table 1. In test medicines, the active compounds were not significantly decreased by the UV irradiation. Naproxen tablets only showed the change of the color from white to yellow, and appearance of two new peaks indicated the generation of its photoproducts (photoproduct 1 and photoproduct 2) in the HPLC analysis (Fig. 1A-B). Retention times were $20.7 \mathrm{~min}$ (naproxen), $17.4 \mathrm{~min}$ (photoproduct 1) and $12.8 \mathrm{~min}$ (photoproduct 2). It is suggested that photoproduct 1 and photoproduct 2 have higher polarity than naproxen because of their shorten retention times.

\subsection{The effect of the change of the dosage form of naproxen tablet on the photostability}

The effect of the change of the dosage form of naproxen tablets on the photostability were examined. Residual rates of active compounds of naproxen powder and suspension prepared from naproxen tablets after the UV irradiation for $24 \mathrm{hr}$ were shown in Fig. 2. Active compound of naproxen powder was degraded to $82 \%$ and that of naproxen suspension was degraded to $67 \%$ after the UV irradiation for $24 \mathrm{hr}$ differently from the result of naproxen tablet. In the case of a naproxen solution, naproxen was completely degraded after the UV irradiation for $3 \mathrm{hr}$. These results indicated that the change of the dosage form of naproxen tablet induced the decrease of photostability. The UV irradiation to naproxen powder and suspension induced the generation of peaks of photoproduct 1 and photoproduct 2 . The generation of photoproduct 1 and photoproduct 2 from the naproxen suspension was more significant than from the naproxen powder in the HPLC analysis (Fig. 1C-F). In our experiments, the control sample of naproxen powder and suspension was unchanged indicating that the degradation of naproxen was derived from the photodegradation only.

There are previous reports indicating that the UV irradiation and the sunlight irradiation induced the photodegradation of various medicines in the solution [8-11]. Our previous reports also showed the various medicines were degraded by the UV irradiation and degradation rate was higher at shorter wavelength [7-8] due to the energy of radiation is derived from the wavelength of the UV. Also, the chemical structure of medicines has a contribution on their photostability. Nifedipine and barnidipine, which have dihydropyridine ring, are tending to be photo-oxidized to pyridine derivatives [12-13], and an ascorbic acid, which has 2,3-enediol moiety, is also tending to be photo-oxidized to dehydroascorbic acid by the UV irradiation [14]. Both the irradiated-UV and the chemical structure, which absorbed the emitted UV, are important factors for the photodegradation of medicines.

\subsection{Structure determination of naproxen photoproducts}

Next, the chemical structure of two naproxen photoproducts were determined using the UV absorption spectrum analysis and NMR analysis. Results of the UV absorption spectrum analysis were shown in Fig. 3. Naproxen showed the maximum absorption at $228 \mathrm{~nm}$. However, the photoproduct 1 showed the maximum absorption at longer region wavelength ( $\lambda \max$ : $242 \mathrm{~nm}$ and $312 \mathrm{~nm}$ ), suggesting that chemical structure of the photoproduct 1 may have a wide conjugating structure 
A.<smiles>COc1ccc2cc([C@@H](C)C(=O)O)ccc2c1</smiles>

B.<smiles>COc1ccc2cc(C(C)=O)ccc2c1</smiles>

C.<smiles>COc1ccc2cc(C(C)O)ccc2c1</smiles>

Fig. 4. Chemical structure of (A) naproxen, (B) photoproduct 1 (2-acetyl-6-methoxy-naphthalene) and (C) photoproduct 2 (1-(6-methoxy-2-naphthyl)ethanol).

compared to naproxen. On the other hand, the UV absorption spectrum of the photoproduct 2 was the same as to that of naproxen ( $\lambda$ max: $229 \mathrm{~nm}$ ), indicating that the chemical structure of the photoproduct 2 may be similar to naproxen.

The chemical structure of naproxen was shown in Fig. 4A. The peak of proton derived from carboxylic acid of naproxen was not detected in this experiment. Results of NMR analysis showed that the photoproduct 1 and the photoproduct 2 had a naphthalene backbone as the same to naproxen. Based on the comparison of ${ }^{1} \mathrm{H}-\mathrm{NMR}$ and ${ }^{13} \mathrm{C}-\mathrm{NMR}$ spectra between naproxen and the photoproduct 1 or 2 , the numbers of proton and carbon of the photoproduct 1 were less than naproxen, and peaks of protons and carbons derived from the aryl group were shifted to the low magnetic field side. In the case of the photoproduct 2, compared to naproxen, the number of carbons was decreased but the number of protons was increased compared to naproxen differently from the results of the photoproduct 1, and peaks derived from the aryl group were shifted to the low magnetic field side as the same to the photoproduct 1, whereas the peaks derived from the methoxy group of both photoproducts were unchanged. These results suggested the decarboxylation of naproxen followed by the addition of oxygen to the aryl group as a carbonyl oxygen or a hydroxyl group, which has an electron with-drawing potency, induced by the UV irradiation.
Considering from the chemical shift and coupling pattern of ${ }^{1} \mathrm{H}-\mathrm{NMR},{ }^{13} \mathrm{C}-\mathrm{NMR}$ spectra and results of two-dimensional NMR, the photoproduct 1 was determined as 2-acetyl-6-methoxy-naphthalene, and the photoproduct 2 was determined as 1-(6-methoxy-2-naphthyl)ethanol (Fig. 4B-C). 2-Acetyl-6-methoxy-naphthalene has a wide conjugating structure compared to naproxen and 1-(6-methoxy-2-naphthyl)ethanol has a similar chemical structure with naproxen, which are corresponded to results of the UV absorption spectrum analysis (Fig. 3). The putative photodegradation pathway of naproxen is shown in Fig. 5. It is speculated that naproxen was excited by the UV irradiation (mainly at $365 \mathrm{~nm}$ ) and its carboxylic acid group was eliminated, followed by the forming of the intermediate product of a secondary carbon anion derivative. After that, this intermediate reacted with an oxygen molecule in the air to form 2-acetyl-6-methoxy-naphthalene or 1-(6-methoxy-2-naphthyl)ethanol. It is reported that the molar absorptivity at $254 \mathrm{~nm}$ of naproxen $\left(4.18 \times 10^{3}\right.$ $\left.\mathrm{M}^{-1} \mathrm{~cm}^{-1}\right)$ and 1-(6-methoxy-2-naphthyl)ethanol (4.35 x $10^{3}$ $\mathrm{M}^{-1} \mathrm{~cm}^{-1}$ ) were smaller than that of 2-acetyl-6-methoxy-naphthalene (3.06 x $\left.10^{4} \mathrm{M}^{-1} \mathrm{~cm}^{-1}\right)$ [15]. From the peak area and the molar absorptivities of two photoproducts, generation rates of them were at the same levels.

There are several reports showing that the UV irradiation induced the photo-conversion of naproxen to various photoproducts including 2-acetyl-6-methoxy-naphthalene and 1-(6-methoxy-2-naphthyl)ethanol [16-19]. These reports utilized naproxen solution, which is dissolved in methanol, acetonitrile and mixture of water and organic solvent, and low-pressure mercury lamp emitting at $254 \mathrm{~nm}$, indicating that the photochemical reaction of naproxen was more significant compared to our experiments because the UV irradiation with high energy and dissolved naproxen in the solution enabled the photodegradation of naproxen to be faster. In this study, residual naproxen was detected in the UV-irradiated tablet, powder and suspension after $24 \mathrm{hr}$ although, in the previous report, naproxen was degraded completely within $1 \mathrm{hr}$ [16]. This is the first study showing the photodegradation of naproxen in the medicine by the
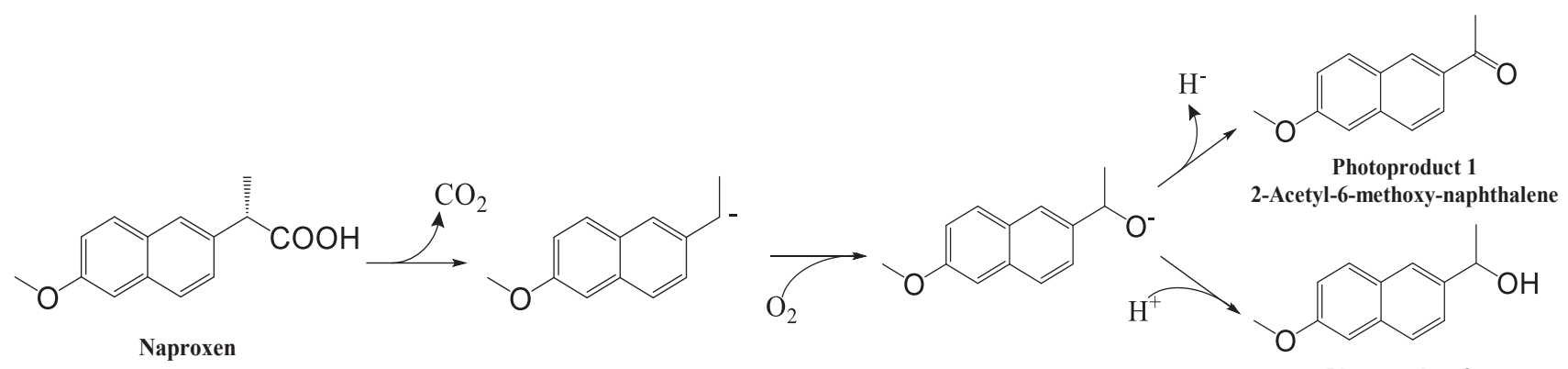

Photoproduct 2

1-(6-Methoxy-2-naphthyl)ethanol

Fig. 5. Proposed pathway of the photodegradation of naproxen to photoproduct 1 and photoproduct 2 . 
UV irradiation at longer wavelength.

There are some reports showing effects of the photodegradation on the biological activities of irradiated medicines [20-24]. In the case of naproxen, some photoproducts of it were more ecotoxic than naproxen, both in acute and chronic conditions [17,25]. These information indicated the importance of avoiding the naproxen tablet, its powder and suspension from the UV irradiation on account of their harmful potential.

\section{Conclusion}

In conclusion, we have shown that the change of the dosage form of naproxen tablet induced the decrease of photostability of it. Furthermore, two photoproducts were determined from the UV-irradiated naproxen medicine. Photostability of medicine is the important factor for the quality and quantity of it, so further studies focused on various medicines are required because the information about the photostability of them, especially in the solid-form, were not clarified.

\section{Supporting information}

${ }^{1} \mathrm{H}-\mathrm{NMR}$ and ${ }^{13} \mathrm{C}-\mathrm{NMR}$ spectrum, two-dimensional NMR spectrum of naproxen and those of the two photoproducts are available via the WEB at http://chromsoc.jp/Journal/SI.html.

\section{Acknowledgements}

This work was supported by the grant of Yasuda Women's University in 2019.

\section{References}

[1] ICH. Fed. Regist. 2003, 68, 65717-65718.

[2] ICH. Fed. Regist. 1996, 62, 27115-27122.

[3] Marinkovic, V. D.; Agbaba, D.; Karljikovic-Rajic, K.; Vladimirov, S.; Nedeljkovic, J. M. J. Pharm. Biomed. Anal. 2003, 32, 929-935.

[4] Ioele, G.; Oliverio, F.; Andreu, I.; De Luca, M.; Miranda, M. A.; Rango, G. J Photochem Photobiol A: Chem. 2010, 215, 205-213.

[5] DellaGreca, M.; Iesce, M. R.; Previtera, L.; Rubino, M.; Temussi, F. Environ. Chem. Lett. 2004, 2, 155-158.

[6] Musa, K. A.; Eriksson, L. A. Phys. Chem. Chem. Phys. 2009, 11, 4601-4610.

[7] Kawabata, K.; Akimoto, S.; Nishi, H. Chromatography 2018, 39, 139-146.

[8] Kawabata, K; Sugihara, K.; Sanoh, S.; Kitamura, S.; Ohta, S. J. Toxicol. Sci. 2013, 38, 215-223.

[9] Yamamoto, H.; Nakamura, Y.; Moriguchi, S.; Nakamura, Y.; Honda, Y.; Tamura, I.; Hirata, Y.; Hayashi, A.; Sekizawa, J. Water Res. 2009, 43, 351-362.
[10] Kin, I.; Tanaka, H. Environ. Int. 2009, 35, 793-802.

[11] Tong, L.; Eichhorn, P.; Perez, S.; Wang, Y.; Barcelo, D. Chemosphere 2011, 83, 340-348.

[12] Majeed, I. A.; Murray, W. J.; Newton, D. W.; Othman, S.; Al-Turk, W. A. J. Pharm. Pharmacol. 1987, 39, 1044-1046.

[13] Nunez-Vergara, L. J.; Bollo, S.; Fuentealba, J.; Sturm, J. C.; Squella, J. A. Pharm. Res. 2002, 19, 522-529.

[14] Mori, Y.; Nishiyama, M.; Morotomi, S. J. Jpn. Soc. Nutr. Food. Sci. 1969, 22, 12-16.

[15] Marotta, R.; Spasiano, D.; Somma, I. D.; Andreozzi, R. Water Res. 2013, 47, 373-383.

[16] Moore, D. E.; Chappuis, P. P.; Photochem. Photobiol. 1988, 47, 173-180.

[17] Ishidori, M.; Lavorgna, M.; Nardelli, A.; Parrella, A.; Previtera, L.; Rubino, M. Sci. Total Environ. 2005, 348, 93-101.

[18] Tu, N.; Liu, Y.; Li, R.; Lv, W.; Liu, G.; Ma, D. Chemosphere 2019, 227, 142-150.

[19] Liang, R.; Sun, S-. S.; Huang, G.; Li, M-. D. Chem. Res. Toxicol. 2019, 32, 613-620.

[20] Ma, D.; Liu, G.; Lv, W.; Yao, K.; Zhang, X.; Xiao, H. Environ. Sci. Pollut. Res. Int. 2014, 21, 7797-7804.

[21] Isidori, M.; Nardelli, A.; Parrella, A.; Pascarella, L.; Previtera, L. Chemosphere 2006, 63, 785-793.

[22] Liu, Q. T.; Williams, T. D.; Cumming, R. I.; Holm, G.; Hetheridge, M. J.; Murray-Smith, R. Environ. Toxicol. Chem. 2009, 28, 2622-2631.

[23] Trovo, A. G.; Nogueira, R. F. P.; Aguera, A.; Sirtori, C.; Fernandez-Alba, A. R. Chemosphere 2009, 77, 1292-1298.

[24] Li, J.; Ma, L. Y.; Li, L. S.; Xu, L. Environ. Toxicol. Chem. 2017, 36, 3232-3239.

[25] Della Greca, M.; Brigante, M.; Isidori, M.; Nardelli, A.; Previtera, L.; Rubino, M.; Temussi, F. Environ. Chem. Lett. 2003, 1, 237-241. 